risk of dementia; OR in a multivariate logistic regression was 2.91 (95\% CI 1.37-6.16). More social contact at baseline was associated with lower risk of incident dementia; OR in the multivariate analysis was 0.87 (95\% CI $0.78-0.97$ ). There were no significant associations between vascular risk factors or comorbidities and dementia risk. Conclusions: Presence of depression at baseline seems to be associated with higher risk of incident dementia after 5 years, whereas more frequent social contacts seem to be associated with lower risk. This highlights the possible role of psychosocial factors in dementia development among the oldest old. Further research on risk and protective factors is needed for these age groups.

\section{O5-05-03 VITAMIN D AND INCIDENT DEMENTIA IN THE CARDIOVASCULAR HEALTH COGNITION STUDY}

Maya Soni ${ }^{1}$, Iain Lang ${ }^{2}$, Kenneth Langa ${ }^{3}$, Paolo Chavez ${ }^{4}$, Cedric Annweiler ${ }^{5}$, Linda Fried ${ }^{6}$, Bryan Kestenbaum ${ }^{7}$, Oscar Lopez ${ }^{8}$, David Llewellyn ${ }^{1},{ }^{1}$ University of Exeter, Exeter, United Kingdom; ${ }^{2}$ Peninsula College of Medicine and Dentistry, Exeter, United Kingdom; ${ }^{3}$ University of Michigan, Ann Arbor, Michigan, United States; ${ }^{4}$ Florida International University, Miami, Florida, United States; ${ }^{5}$ Angers University Hospital, Angers University Hospital, New York, United States; ${ }^{6}$ Columbia University, New York, New York, United States; ${ }^{7}$ University of Washington, Seattle, Washington, United States; ${ }^{8}$ University of Pittsburgh, Pittsburgh, Pennsylvania, United States. Contact e-mail: maya.soni@pcmd.ac.uk

Background: Recent meta-analyses establish that serum vitamin D levels are lower in Alzheimer's disease patients and low levels are associated with poorer cognitive function. Preliminary prospective studies also suggest that low vitamin D levels may be associated with greater cognitive decline, though the link with incident dementia is largely unknown. Methods: We determined whether low levels of serum 25-hydroxyvitamin D $(25(\mathrm{OH}) \mathrm{D})$ were associated with an increased risk of incident dementia in the population-based Cardiovascular Health Cognition Study conducted in the US. A total of 1,653 ambulatory adults 64 years or older and free from dementia and cardiovascular disease provided blood samples in 1992-1993 and were assessed for dementia over a mean of 5.6 years follow-up $(\mathrm{SD}=1.2)$. A total of 171 cases of incident a ll-cause dementia were identified according to Diagnostic and Statistical Manual of Mental Disorders, Fourth Edition (DSMIV) criteria by a committee of neurologists and psychiatrists using cognitive tests, neurological examinations, medical records, physician questionnaires, and proxy interviews. Circulating serum $25(\mathrm{OH}) \mathrm{D}$ concentrations were measured using mass spectrometry. Cox proportional hazard models were used to investigate the relationship between vitamin $\mathrm{D}$ and incident dementia adjusting for age, education, season of blood draw, gender, body mass index, depressive symptoms, alcohol consumption, and hypertension. Results: The multivariate adjusted hazard ratio (95\% confidence interval [CI]) of incident dementia in participants who were severely serum $25(\mathrm{OH}) \mathrm{D}$ deficient $(<25 \mathrm{nmol} / \mathrm{L})$, deficient $(25$ to $<50 \mathrm{nmol} / \mathrm{L})$, and insufficient ( 50 to $<75 \mathrm{nmol} / \mathrm{L}$ ) in comparison with those with sufficient levels ( $\geq 75 \mathrm{nmol} / \mathrm{L}$ ) was 1.97 (95\% CI, 1.03 to 3.79 ), 1.33 (95\% CI, 0.86 to $2.04)$ and 0.80 ( 0.54 to 1.17 ) respectively ( $\mathrm{p}$ for linear trend across groups $=0.04$ ). This corresponds to an unadjusted incidence of dementia of $18.8 \%$ in severely $25(\mathrm{OH}) \mathrm{D}$ deficient participants in comparison with 9.9\% in those sufficient. Conclusions: Severe vitamin D deficiency was associated with double the risk of dementia in the elderly US population over a mean of 5.6 years follow-up. Randomized controlled trials are needed to establish whether supplementing older adults with low baseline vitamin D levels is an effective disease modifying intervention for dementia.

\section{O5-05-04 SELF-REPORTED HEAD INJURY AND RISK OF COGNITIVE IMPAIRMENT AND ALZHEIMER'S- TYPE PATHOLOGY IN A LONGITUDINAL ALZHEIMER'S DISEASE CENTER COHORT}

Erin Abner ${ }^{1}$, Peter Nelson ${ }^{1}$, Frederick Schmitt ${ }^{1}$, Steven Browning ${ }^{1}$, David Fardo ${ }^{2}$, Lijie Wan ${ }^{1}$, Gregory Jicha ${ }^{1}$, Gregory Cooper ${ }^{3}$, Charles Smith ${ }^{4}$, Allison Caban-Holt ${ }^{1}$, Linda Van Eldik ${ }^{1}$, Richard Kryscio ${ }^{1}$, ${ }^{1}$ University of Kentucky, Lexington, Kentucky, United States; ${ }^{2}$ College of Public Health, Lexington, Kentucky, United States; ${ }^{3}$ Baptist Neurology
Center, Lexington, Kentucky, United States; ${ }^{4} U K$ MRISC, Lexington, Kentucky,United States. Contact e-mail: erin.abner@uky.edu

Background: The effect of head injury on dementia risk remains incompletely understood. To date, longitudinal studies of premorbid head injury and late-life dementia have been largely limited to antemortem clinical status, but have not been pathologically verified. Methods: Clinical and neuropathological data from participants enrolled in a longitudinal study of aging and cognition at the University of Kentucky Alzheimer's Disease Center $(n=649)$ were analyzed to assess the long-term effects of self-reported head injury using a multistate Markov chain, a statistical model capable of handling competing risks (i.e., death without dementia and dropout without dementia). MANCOVA models were constructed to assess whether history self-reported head injury was associated with increased AD-type pathological burden in subjects with quantitative neuropathologic data $(n=238)$. Results: The effect of self-reported head injury on clinical state depends on age: for a one-year increase in age, the OR for transition from a transient state to clinical MCI for participants with a history of head injury is 1.21 (95\% CI: 1.15-3.56). Similarly, for a one-year increase in age, the OR for transition from MCI to dementia for participants with a history of head injury is 1.34 (95\% CI: 1.11-1.61). The odds of having AD pathology sufficient for an $\mathrm{AD}$ diagnosis are significantly increased for men with a history of head injury $(\mathrm{OR}=1.47,95 \% \mathrm{CI}: 1.03-2.09)$ but not for women $(\mathrm{OR}=1.18$, 95\% CI: $0.83-1.68)$. Self-reported head injury, without respect to age, is a significant risk for the one-step transition from a clinically unimpaired state to death without dementia (OR $=1.54,95 \%$ CI: $1.12-2.13$ ). MANCOVA revealed that men with a history of head injury have significantly higher mean diffuse and neuritic plaque counts in the neocortex and entorhinal cortex compared to men without. No cases of chronic traumatic encephalopathy were observed in the sample. Conclusions: These results support prior work identifying earlier onset and increased risk of cognitive impairment with head injury and also demonstrate an increased risk of mortality. Moreover, for men, whose injuries were more severe than the women in this sample, these injuries appear to also increase the burden of AD-type amyloid plaques.

\section{5-05-05 THE EFFECT OF AGE OF STARTING A CONTACT SPORT ON SUBSEQUENT BRAIN VOLUMES}

Sierra Simmons ${ }^{1}$, Charles Bernick ${ }^{2}$, Sarah Banks ${ }^{1}$, Nancy Obuchowski ${ }^{1}$, ${ }^{1}$ Cleveland Clinic, Las Vegas, Nevada, United States; ${ }^{2}$ Cleveland Clinic Lou Ruvo Center for Brain Health, Las Vegas, Nevada, United States. Contact e-mail: scsimmons@medicine.nevada.edu

Background: Repetitive head trauma is a risk factor for Chronic Traumatic Encephalopathy but it is unclear what factors modify the risk. This study examines how age at which one begins exposure to head trauma interacts with amount of exposure to head trauma on MRI determined caudate volume. Methods: 239 professional fighters ( boxers, mixed martial arts fighters) participating in the Professional Fighters Brain Health Study were included for analysis. Subjects underwent MRI brain imaging with automated volumetric measurements of brain structures. Age of beginning competitive fighting was obtained from self-report. Professional fighting record, including years of fighting, number of fights and fights per year were obtained from published records. Repeated measures ANOVA was performed to test the association between the fight exposure variables and caudate volumes, with age started fighting added as an independent predictor. All analyses were adjusted for age, education, race, and intracranial volume. Results: The interaction between the number of professional fights and age started fighting had a significant effect on caudate volume. As the number of professional fights increases, the caudate volume decreases, but this effect is mediated by the age started fighting $(\mathrm{p}=0.048)$. For fighters who started fighting early $(<15$ years old $)$, there is an estimated $0.37 \%$ reduction in caudate volume per professional fight, whereas for fighters who started fighting later $(>15$ $\mathrm{y} / \mathrm{o}$ ), there is an estimated $0.10 \%$ reduction in caudate volume per professional fight. Conclusions: Age of starting a sport which involves blows to the head may modify the effects of subsequent repetitive head trauma on brain volumes. Prospective follow up of this cohort may help determine if 